Case Report

\title{
Colonoscopic Splenic Injury: A Simplified Radiologic Approach
}

\author{
Tara Chen, Qiu Tong, and Alexander Kurchin \\ Department of Surgery, Rochester General Hospital, Rochester, NY, USA \\ Correspondence should be addressed to Qiu Tong; tongq6@gmail.com
}

Received 1 August 2016; Accepted 27 November 2016

Academic Editor: Tetsuo Hirata

Copyright (C) 2016 Tara Chen et al. This is an open access article distributed under the Creative Commons Attribution License, which permits unrestricted use, distribution, and reproduction in any medium, provided the original work is properly cited.

Colonoscopy is a commonly performed procedure for diagnosis and treatment of large bowel diseases. Recognized complications include bleeding and perforation. Splenic injury during colonoscopy is a rare complication. We report a case of a 73-year-old woman who presented with left-sided abdominal pain after colonoscopy with finding of splenic injury on CT scan. She was managed conservatively. We discuss the diagnostic and therapeutic approach to colonoscopic splenic injury.

\section{Introduction}

Approximately 14 million colonoscopies are performed in the US every year [1]. It is a safe procedure, with the main complications being bleeding (1-2\%) and colonic perforation (0.1-0.2\%) [2]. A rare and potentially fatal complication is splenic injury due to manipulation of the colonoscope through the large bowel.

When a patient presents with abdominal pain following colonoscopy, the main concern is that of colonic perforation [3]. CT scan of the abdomen and pelvis is highly sensitive for diagnosing colonic perforation [3]. Splenic injury is usually an unexpected finding of the test. Treatment is based on the hemodynamic status of the patient and on the degree of splenic injury seen on the CT scan [4].

\section{Case Presentation}

A 73-year-old woman underwent a screening colonoscopy that was initially reported as uneventful. Her medical history included atrial fibrillation treated successfully with radioablation. She was not on anticoagulation at the time of her exam. In her late 40s she underwent a total hysterectomy. Her medications included aspirin $81 \mathrm{mg}$ daily.

Colonoscopy was done under moderate sedation with $75 \mathrm{mcg}$ of fentanyl and $5 \mathrm{mg}$ of midazolam given intravenously. The colonoscope was advanced to the cecum without difficulty. The exam was normal except for pandiverticulosis. The nurses' notes indicated that the patient had mild left-sided abdominal pain shortly after the procedure. This improved after passing gas and she was considered suitable for discharge.

Five hours after the procedure she called the endoscopist reporting increasing left-sided abdominal pain. There was no associated nausea, vomiting, fever, chills, dyspnea, or left shoulder pain. She was reevaluated in the endoscopist's office. On exam her vital signs were normal and there was moderate left-sided abdominal tenderness. There was no rebound or guarding. Concern for colonic perforation prompted a CT scan of the abdomen and pelvis, done without intravenous contrast. The study revealed high density perisplenic fluid consistent with blood, tracking in the left paracolic gutter (Figure 1), with no evidence of parenchymal damage. These findings indicated a grade 1 splenic injury (Table 1) and the patient was admitted for observation. She was managed conservatively with intravenous fluids. A modest overnight drop in hematocrit from $41 \%$ to $35 \%$ was noted, presumed to be partially related to hemodilution caused by the IV fluids. The next morning, the patient remained hemodynamically stable and her abdominal tenderness was much improved. She was discharged and seen in the office the following day with no further complaints of abdominal pain and no tenderness upon abdominal exam.

\section{Discussion}

Splenic injury is a rare complication of colonoscopy and is usually not a diagnostic consideration in patients who present 
TABLE 1: The American Association of Surgeons for Trauma (AAST). Splenic Injury Grading Scale (2008).

\begin{tabular}{|c|c|c|}
\hline Grade & Injury & Criteria \\
\hline \multirow{2}{*}{ I } & Hematoma & Subcapsular, $<10 \%$ of surface area \\
\hline & Laceration & Capsular tear, $<1 \mathrm{~cm}$ parenchymal depth \\
\hline \multirow[t]{2}{*}{ II } & Hematoma & $\begin{array}{l}\text { Subcapsular, } 10-50 \% \text { of surface area; } \\
\text { intraparenchymal, }<5 \mathrm{~cm} \text { diameter }\end{array}$ \\
\hline & Laceration & $\begin{array}{l}\text { Subcapsular, } 1 \mathrm{~cm} \text { to } 3 \mathrm{~cm} \text { parenchymal depth that } \\
\text { does not involve a trabecular vessel }\end{array}$ \\
\hline \multirow[t]{2}{*}{ III } & Hematoma & $\begin{array}{c}\text { Subcapsular, }>50 \% \text { of surface area or expanding; } \\
\text { ruptured subcapsular or parenchymal hematoma; } \\
\text { intraparenchymal hematoma, } \geq 5 \mathrm{~cm} \text { diameter or } \\
\text { expanding }\end{array}$ \\
\hline & Laceration & $\begin{array}{c}>3 \mathrm{~cm} \text { parenchymal depth or involving trabecular } \\
\text { vessels }\end{array}$ \\
\hline IV & Laceration & $\begin{array}{l}\text { Laceration involving segmental or hilar vessels } \\
\text { producing major devascularization of }>25 \% \text { of the } \\
\text { spleen }\end{array}$ \\
\hline \multirow[b]{2}{*}{$\mathrm{V}$} & Hematoma & Completely shattered spleen \\
\hline & Laceration & $\begin{array}{c}\text { Hilar vascular injury that devascularizes the } \\
\text { spleen }\end{array}$ \\
\hline
\end{tabular}

* Advance one grade for multiple injuries, up to grade III.

Reprinted from [4], with permission from Elsevier.

License number 3917700781230.

Source: http://www.sciencedirect.com/science/article/pii/S1072751508011010.

with abdominal pain after the procedure. Since the first case report by Wherry et al., in 1974, around 100 cases have been reported [5-7]. The incidence has been estimated to be in the range of $0.00005 \%$ to $0.017 \%$ [8]. A review of ICD-9 coding for the combination of colonoscopy and splenic injury showed 437 cases out of 2,654,456 colonoscopies performed between the years 2000 and 2007, yielding a rate of $0.016 \%$ or about 1 out of 6000 [8]. The true incidence is likely higher due to underreporting resulting from a reluctance to publish information on procedural complications and lack of clear definition of colonoscopic splenic injury [7, 8]. Furthermore, mild cases may resolve before a diagnosis is made or may be misdiagnosed as nonspecific postcolonoscopy discomfort [7]. This complication has a female preponderance, with approximately $75 \%$ of cases being diagnosed in women $[7,9]$.

The mechanism of injury is presumably due to downwards traction on the splenocolic ligament or on adhesions around the spleen during colonoscopic manipulation causing a tear in the splenic capsule $[2,6,8-10]$. Capsular tear or parenchymal injury may also result from direct trauma inflicted on the spleen as the colonoscope passes through the splenic flexure [2, 8-10]. While anticoagulation and antiplatelet therapy do not, per se, cause splenic injury, they are likely to increase the degree of bleeding to a level that will be symptomatic enough to lead to a diagnosis [7]. The presence of splenomegaly has not been described as a contributing factor, but we may assume that an enlarged spleen will be more prone to injury, as reported in the trauma literature [11].

Patients with splenic injury during colonoscopy usually present within 24 to 48 hours, but presentation can be delayed

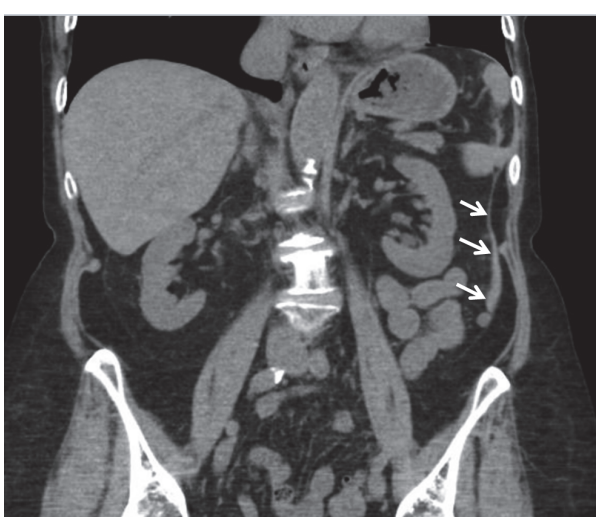

FIGURE 1: CT abdomen and pelvis without contrast. Coronal view showing small amount of high density perisplenic fluid (white arrows) tracking in the left paracolic gutter and extending into the pelvis.

for up to 10 days after the procedure $[2,7,8,10]$. The most common complaint is left upper quadrant abdominal pain [8]. Additional symptoms include dizziness, palpitations, referred left shoulder pain from diaphragm irritation (Kehr's sign), and diffuse abdominal discomfort [8]. On physical examination patients may have localized left upper quadrant tenderness with or without signs of peritoneal irritation including guarding and rebound [12]. However, these signs and symptoms are nonspecific and the diagnosis of splenic injury is almost never made based on history and exam alone. 
The majority of cases are diagnosed by CT scan or at the time of laparotomy [7, 9, 13].

CT of the abdomen is the most reliable diagnostic tool for stable patients $[4,9,13]$. Findings suggestive of splenic injury include hemoperitoneum, hypodensity of the spleen, and contrast blush indicating extravasation of blood [13]. Although CT scan without intravenous contrast does not demonstrate parenchymal injury as well as a study with contrast, it is still quite sensitive. A retrospective analysis concluded that CT without contrast has a sensitivity of $93 \%$ in detecting splenic injury based on findings of a perisplenic hematoma or free abdominal fluid and strong clinical suspicion [13]. Furthermore, CT scan without contrast will quickly and accurately diagnose colonic perforation, which is the main concern when evaluating postcolonoscopy pain. The advantages of a study without contrast include the rapidity of the procedure and the ease of performing the test in an outpatient facility. It eliminates the need for IV access, checking creatinine, the risk of contrast induced nephropathy, and the concern for contrast allergy. We recommend, as injury is diagnosed on a CT scan without contrast, usually as an unexpected finding, not to repeat the test with contrast on a routine basis. In the few patients in whom more anatomic detail or evaluation of active bleeding is needed, the study can be repeated with contrast [13].

The American Association of Surgeons for Trauma (AAST) Splenic Injury Grading Scale is used to grade splenic injuries based on CT scan findings (Table 1) [4]. Treatment is based on the patient's clinical condition and the extent of splenic injury. There are three treatment options described in the literature: nonoperative management with fluid resuscitation and hemodynamic monitoring, interventional radiologic splenic artery embolization, and surgery $[2,6-8,10]$. Nonoperative treatment is the preferred choice, avoiding major surgery and preserving splenic immune function [12, 14]. Nonoperative management of splenic injury with fluid resuscitation, bed rest, and hemodynamic monitoring is more likely to succeed in patients with AAST grade I or II injury who are hemodynamically stable, lack peritoneal findings on exam, are younger than 55 years of age, and require little to no transfusion $[12,15,16]$.

An estimated $85 \%$ of patients with blunt splenic injury in the past decade were managed nonoperatively [15]. In patients who fail this initial management and have ongoing bleeding but are hemodynamically stable, splenic artery embolization, which is highly successful in patients in blunt trauma, is the next step $[16,17]$. The procedure has decreased the failure rate of nonoperative management from $12-13 \%$ to $2-3 \%$ [15]. The procedure appears to be safe, both in the elderly population with comorbidities and in patients who take medication that increases the risk of bleeding [15].

Surgical management is indicated in patients who fail conservative treatment and splenic artery embolization. The standard approach is open exploration. Laparoscopic surgery, which may be technically more demanding and more time consuming, is reserved for select patients with minimal hemorrhage and who are hemodynamically stable $[18,19]$. Attempts at splenic salvage include splenorrhaphy and partial splenectomy. Splenectomy is indicated when all other management procedures have failed. Mortality from spleen injury is reported to be $5 \%$, with a worse prognosis in patients who are diagnosed later [12].

\section{Conclusion}

In patients presenting with abdominal pain following colonoscopy, perforation is the primary concern. CT without contrast is recommended as the first diagnostic test. Splenic injury is so rare that it is almost never considered until diagnosis by CT imaging or laparotomy. This test, done without contrast, is sufficient to rule out colonic perforation and for evaluation of most splenic injuries. The scan can be repeated with IV contrast if necessary to obtain more anatomic information or to document active bleeding. Management is initially nonsurgical, which is successful in most patients. If it fails and the patient is hemodynamically stable, splenic artery embolization is the next step. Surgery is indicated, usually by open technique, when the patient is unstable or if bleeding continues after embolization attempt.

\section{Competing Interests}

The authors declare that they have no conflict of interests regarding this paper.

\section{Authors' Contributions}

Both Tara Chen and Qiu Tong contributed to drafting and revising this paper and performing a search of literature. Alexander Kurchin was active in management of the patient and provided case information and thorough guidance in writing this paper.

\section{References}

[1] L. C. Seeff, T. B. Richards, J. A. Shapiro et al., "How many endoscopies are performed for colorectal cancer screening? Results from CDC's survey of endoscopic capacity," Gastroenterology, vol. 127, no. 6, pp. 1670-1677, 2004.

[2] M. Sarhan, A. Ramcharan, and S. Ponnapalli, "Splenic injury after elective colonoscopy," JSLS, vol. 4, article 616, 2009.

[3] S. M. Kavic and M. D. Basson, "Management of complications of colonoscopy," in Surgical Treatment: Evidence-Based and Problem-Oriented, R. G. Holzheimer and J. A. Mannick, Eds., Zuckschwerdt, Munich, Germany, 2001, https://www.ncbi.nlm .nih.gov/books/NBK6945/.

[4] G. Tinkoff, T. J. Esposito, J. Reed et al., "American association for the surgery of trauma organ injury scale I: spleen, liver, and kidney, validation based on the national trauma data bank," Journal of the American College of Surgeons, vol. 207, no. 5, pp. 646-655, 2008.

[5] D. C. Wherry and H. Zehner Jr., "Colonoscopy-fiberoptic endoscopic approach to the colon and polypectomy," The Medical Annals of the District of Columbia, vol. 43, no. 4, pp. 189-192, 1974.

[6] I. M. Brennan, S. Faintuch, and B. Sacks, "Superselective splenic artery embolization for the management of splenic laceration following colonoscopy," Acta Radiologica Short Reports, vol. 3, no. 3, pp. 1-5, 2014. 
[7] G. Piccolo, M. Di Vita, A. Cavallaro et al., "Presentation and management of splenic injury after colonoscopy: a systematic review," Surgical Laparoscopy, Endoscopy and Percutaneous Techniques, vol. 24, no. 2, pp. 95-102, 2014.

[8] S. Singla, D. Keller, P. Thirunavukarasu et al., "Splenic injury during colonoscopy-a complication that warrants urgent attention," Journal of Gastrointestinal Surgery, vol. 16, no. 6, pp. 1225-1234, 2012.

[9] J. R. A. Skipworth, D. A. Raptis, J. S. Rawal et al., "Splenic injury following colonoscopy - an underdiagnosed, but soon to increase, phenomenon?" Annals of the Royal College of Surgeons of England, vol. 91, no. 4, pp. W6-W11, 2009.

[10] M. A. Zappa, A. Aiolfi, I. Antonini, C. D. Musolino, and A. Porta, "Splenic rupture following colonoscopy: case report and literature review," International Journal of Surgery Case Reports, vol. 21, pp. 118-120, 2016.

[11] J. J. Hallman, K. J. Brasel, N. Yoganandan, and F. A. Pintar, "Splenic trauma as an adverse effect of torso-protecting side airbags: biomechanical and case evidence," Annals of Advances in Automotive Medicine, vol. 53, pp. 13-24, 2009.

[12] M. Beuran, I. Gheju, M. D. Venter, R. C. Marian, and R. Smarandache, "Non-operative management of splenic trauma," Journal of Medicine and Life, vol. 5, no. 1, pp. 47-58, 2012.

[13] D. R. Murken, J. J. Weis, G. C. Hill et al., "Radiographic assessment of splenic injury without contrast: is contrast truly needed?" Surgery, vol. 152, no. 4, pp. 676-684, 2012.

[14] A. Boscak and K. Shanmuganathan, "Splenic trauma: what is new?” Radiologic Clinics of North America, vol. 50, no. 1, pp. 105122, 2012.

[15] A. Corcillo, S. Aellen, T. Zingg, P. Bize, N. Demartines, and A. Denys, "Endovascular treatment of active splenic bleeding after colonoscopy: a systematic review of the literature," CardioVascular and Interventional Radiology, vol. 36, no. 5, pp. 1270-1279, 2013.

[16] A. Raikhlin, M. O. Baerlocher, M. R. Asch, and A. Myers, "Imaging and transcatheter arterial embolization for traumatic splenic injuries: review of the literature," Canadian Journal of Surgery, vol. 51, no. 6, article 464, 2008.

[17] C. H. Van Der Vlies, J. Hoekstra, K. J. Ponsen, J. A. Reekers, O. M. Van Delden, and J. C. Goslings, "Impact of splenic artery embolization on the success rate of nonoperative management for blunt splenic injury," CardioVascular and Interventional Radiology, vol. 35, no. 1, pp. 76-81, 2012.

[18] S. Abunnaja, L. Panait, J. A. Palesty, and S. Macaron, "Laparoscopic splenectomy for traumatic splenic injury after screening colonoscopy," Case Reports in Gastroenterology, vol. 6, no. 3, pp. 624-628, 2012.

[19] A. Carobbi, F. Romagnani, G. Antonelli, and M. Bianchini, "Laparoscopic splenectomy for severe blunt trauma: initial experience of ten consecutive cases with a fast hemostatic technique," Surgical Endoscopy, vol. 24, no. 6, pp. 1325-1330, 2010. 


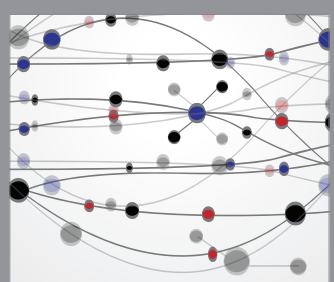

The Scientific World Journal
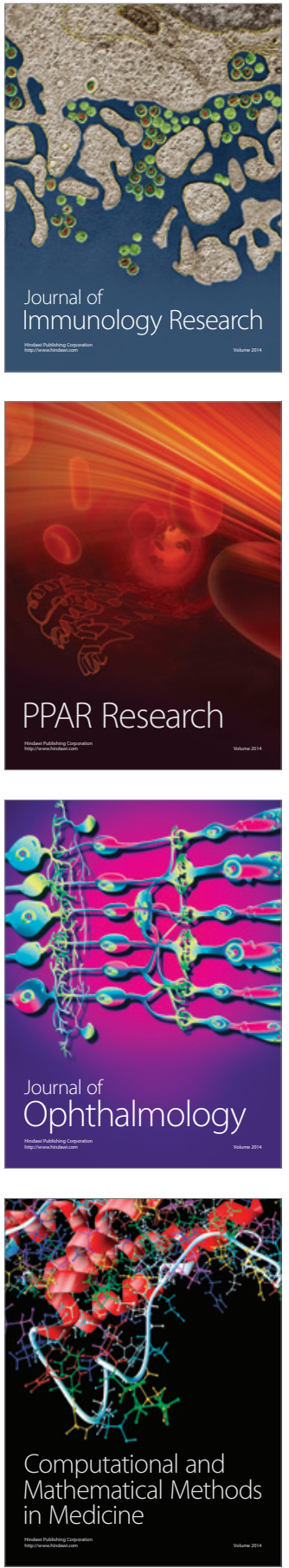

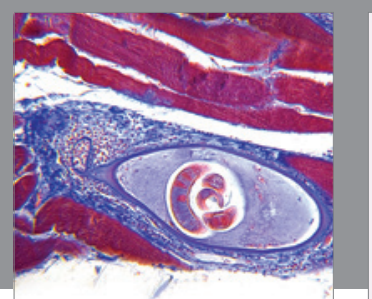

Gastroenterology Research and Practice

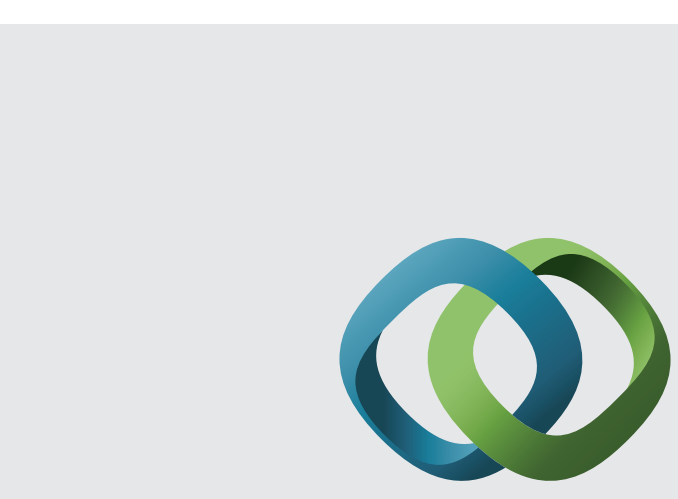

\section{Hindawi}

Submit your manuscripts at

http://www.hindawi.com
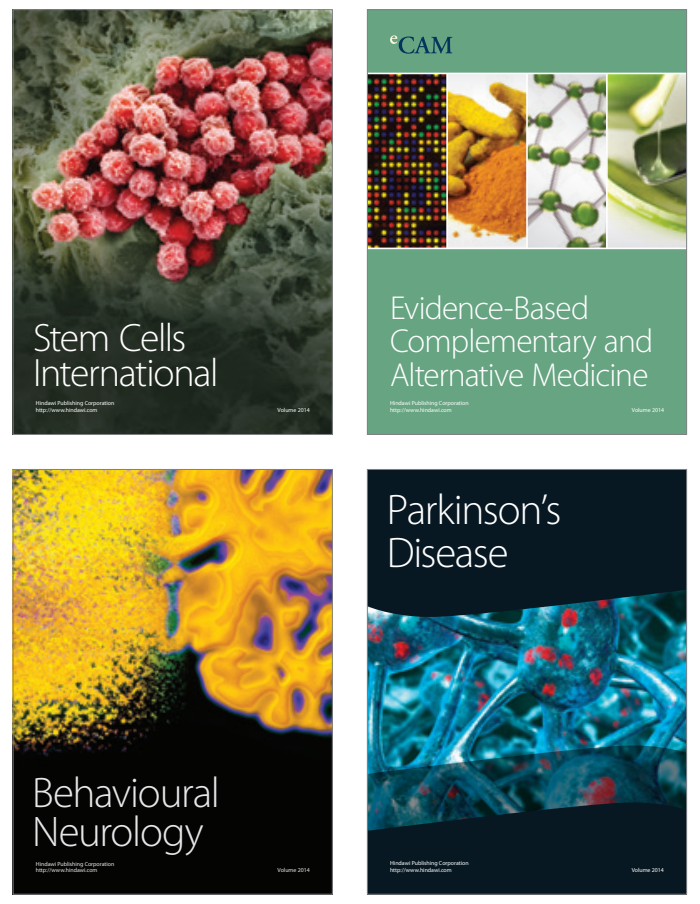
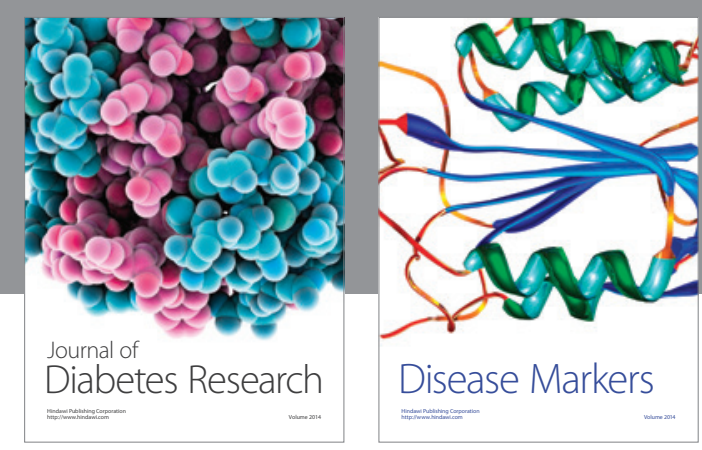

Disease Markers
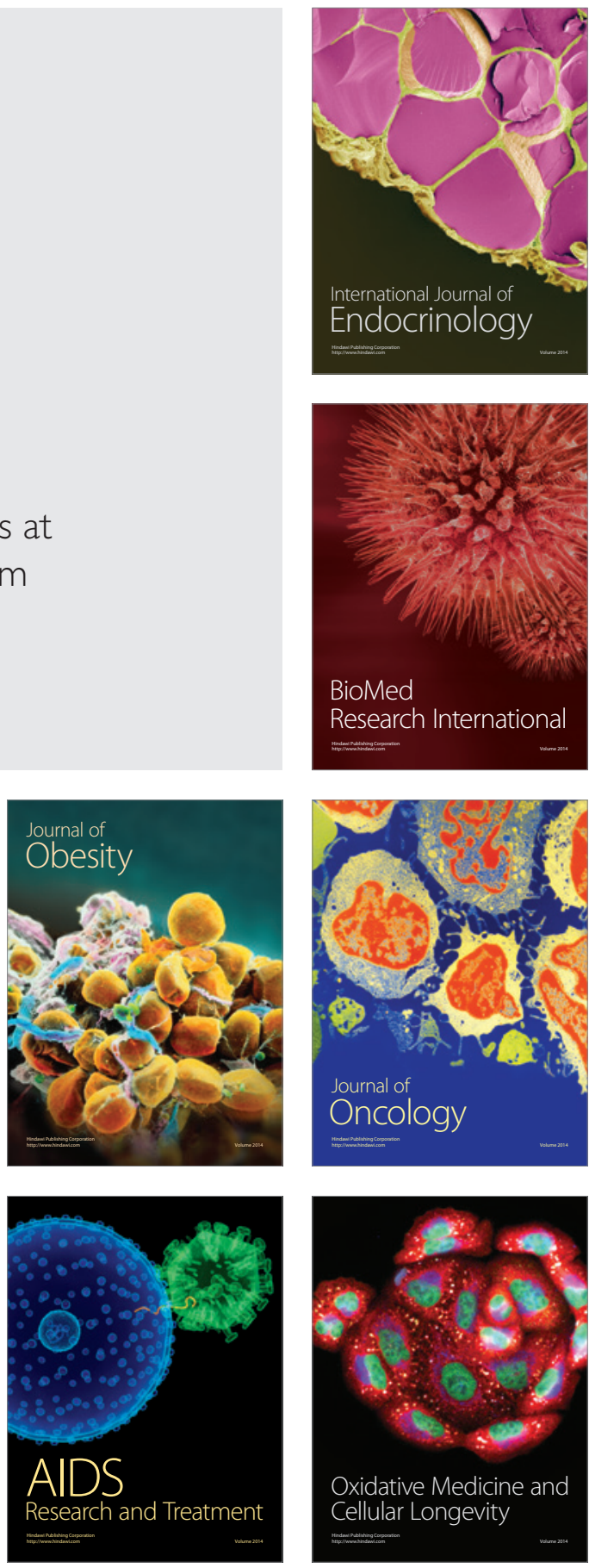